\title{
BMJ Open Treatments for macular oedema following central retinal vein occlusion: systematic review
}

\author{
John A Ford, ${ }^{1}$ Christine Clar, ${ }^{2}$ Noemi Lois, ${ }^{3}$ Samantha Barton, ${ }^{4}$ Sian Thomas, ${ }^{2}$ \\ Rachel Court, ${ }^{2}$ Deepson Shyangdan, ${ }^{2}$ Norman Waugh ${ }^{2}$
}

To cite: Ford JA, Clar C, Lois N, et al. Treatments for macular oedema following central retinal vein occlusion: systematic review. BMJ Open 2014:4:e004120.

doi:10.1136/bmjopen-2013004120

- Prepublication history and additional material for this paper is available online. To view these files please visit the journal online (http://dx.doi.org/10.1136/ bmjopen-2013-004120).

Received 26 September 2013 Revised 3 January 2014 Accepted 14 January 2014

CrossMark

For numbered affiliations see end of article.

Correspondence to Dr John A Ford; john.ford@uea.ac.uk

\section{ABSTRACT}

Objectives: To review systematically the randomised controlled trial (RCT) evidence for treatment of macular oedema due to central retinal vein occlusion (CRVO).

Data sources: MEDLINE, EMBASE, CDSR, DARE, HTA, NHSEED, CENTRAL and meeting abstracts (January 2005 to March 2013).

Study eligibility criteria, participants and interventions: RCTs with at least 12 months of follow-up assessing pharmacological treatments for CRVO were included with no language restrictions.

Study appraisal and synthesis methods: 2 authors screened titles and abstracts and conducted data extracted and Cochrane risk of bias assessment. Meta-analysis was not possible due to lack of comparable studies.

Results: 8 studies (35 articles, 1714 eyes) were included, assessing aflibercept $(\mathrm{n}=2)$, triamcinolone $(n=2)$, bevacizumab $(n=1)$, pegaptanib $(n=1)$, dexamethasone $(n=1)$ and ranibizumab $(n=1)$. In general, bevacizumab, ranibizumab, aflibercept and triamcinolone resulted in clinically significant increases in the proportion of participants with an improvement in visual acuity of $\geq 15$ letters, with $40-60 \%$ gaining $\geq 15$ letters on active drugs, compared to $12-28 \%$ with sham. Results for pegaptanib and dexamethasone were mixed. Steroids were associated with cataract formation and increased intraocular pressure. No overall increase in adverse events was found with bevacizumab, ranibizumab, aflibercept or pegaptanib compared with control. Quality of life was poorly reported. All studies had a low or unclear risk of bias. Limitations: All studies evaluated a relatively short primary follow-up (1 year or less). Most had an unmasked extension phase. There was no head-tohead evidence. The majority of participants included had non-ischaemic CRVO.

\section{Conclusions and implications of key findings:}

Bevacizumab, ranibizumab, aflibercept and triamcinolone appear to be effective in treating macular oedema secondary to CRVO. Long-term data on effectiveness and safety are needed. Head-to-head trials and research to identify 'responders' is needed to help clinicians make the right choices for their patients. Research aimed to improve sight in people with ischaemic CRVO is required.

\section{Strengths and limitations of this study}

- A robust systematic review method was used which only included randomised controlled trials.

- There were no head-to-head trials and there was a lack of long-term data on both effectiveness and safety.

\section{INTRODUCTION}

Central retinal vein occlusion (CRVO) is a vascular disorder of the retina with often catastrophic consequences to vision and quality of life. ${ }^{1}{ }^{2}$ The incidence of CRVO increases with age; most individuals affected are 50 years of age or older. ${ }^{3}$ It has been estimated that there are around 80 new cases of CRVO/million population/year. ${ }^{45}$ Although CRVO most commonly affects one eye, in around $10 \%$ of patients the disease affects both eyes. ${ }^{2}$ Approximately $20 \%$ of patients with CRVO will develop large areas of retinal non-perfusion (ischaemia). ${ }^{6}$ Furthermore, a small proportion (around 8\%) of patients with non-ischaemic CRVO may convert into the ischaemic type during follow-up. ${ }^{6}$ Retinal ischaemia may lead to the development of neovascularisation in the retina, iris or anterior chamber angle. Complications of neovascularisation include vitreous haemorrhage and neovascular glaucoma. ${ }^{6}$ Currently, there is no treatment for ischaemic CRVO other than that aimed at ameliorating the severity of complications, with treatments such as panretinal photocoagulation. Even with the use of current therapies, some eyes with ischaemic CRVO end up blind and painful and, ultimately, enucleation (removal of the eye) is necessary to provide comfort to patients.

Not all people with CRVO will require treatment and macular oedema will resolve in about a third of those with non-ischaemic CRVO. ${ }^{2}{ }^{7}$ However, most will need treatment 
and the number of options has increased in recent years. Laser photocoagulation has been, for many years, the standard therapy for patients with macular oedema secondary to branch retinal vein obstruction (BRVO) ${ }^{8}$ However, laser treatment was not found to be beneficial to those with macular oedema secondary to $\mathrm{CRVO}^{9}$; for these patients, no therapeutic modalities could be offered. Recently, several studies have demonstrated the benefit of antivascular endothelial growth factor (VEGF) therapies and steroids for the management of patients with macular oedema secondary to CRVO. ${ }^{10}{ }^{11}$ Steroids, such as triamcinolone and dexamethasone, have antiinflammatory and antiproliferative attributes (as well as some anti-VEGF effects) and therefore are primarily effective by reducing the oedema of the macula. ${ }^{12}$ Anti-VEGF treatments, such as bevacizumab, ranibizumab, aflibercept and pegaptanib, inhibit vascular endothelial growth factor A. In CRVO there is an increase in vascular endothelial growth factor A which leads to neovascularisation and oedema. ${ }^{13}$ In the UK, National Institute for Health and Care Excellence (NICE) has approved dexamethasone (in the long-acting form, Ozurdex) and ranibizumab (Lucentis) and an appraisal of aflibercept is currently underway. Bevacizumab is also used, but is not licensed for use in the eye; however this is because the manufacturer has never sought a license, preferring to market ranibizumab. Triamcinolone has also been used off license.

An up-to-date review incorporating all drug treatments for macular oedema secondary to CRVO is needed. The purpose of this study is to review systematically the randomised controlled evidence for drug treatments of macular oedema secondary to CRVO.

\section{METHODS}

A systematic review was conducted. The following databases were searched: MEDLINE, MEDLINE In-process, EMBASE (all via OVID); CDSR, DARE, HTA, NHSEED, CENTRAL (all via The Cochrane Library); Science Citation Index and Conference Proceedings Citation Index-Science (via Web of Knowledge). In addition to the bibliographic database searching, supplementary searches were undertaken to look for recent and unpublished studies in the WHO International Clinical Trials Registry Platform and ophthalmology conference websites (American Academy of Ophthalmology, Association for Research in Vision and Ophthalmology from 2010 to 2012).

\section{Search strategy}

An iterative procedure was used to develop two search strategies with input from previous systematic reviews. ${ }^{14}$ 15 The first search strategy was designed to retrieve articles reporting randomised controlled trials (RCTs) or systematic reviews about CRVO published from 2005 onwards (the publication date of the first RCT on triamcinolone in MEDLINE). Terms for retinal vein occlusion were included to ensure identification of articles in which BRVO and CRVO were covered, but were reported separately. The second strategy focussed on retrieving articles where adverse events of relevant pharmacological treatments for CRVO were reported. This second search was limited by condition (age-related macular degeneration (AMD) or RVO), study type (RCTs, SRs or observational studies) and date (published from 2010 onwards). Searches were conducted in March 2013. The strategies used in each database are provided in online supplementary appendix 1. Auto alerts of searches were set up to capture relevant articles published after the dates of the searches.

Reference lists from the included studies and identified systematic reviews were screened.

\section{Inclusion and exclusion criteria}

RCTs were used to assess the clinical effectiveness and adverse events.

Only RCTs examining pharmacological treatment compared with laser treatment, observation, placebo (sham injection) or another pharmacological intervention with at least 12 months follow-up were included. Comparisons of different doses of drugs were not included unless there was an additional comparator group as defined above. Studies including CRVO and BRVO were included provided participants with CRVO were reported as a subgroup. Studies assessing treatments aimed at restoring circulation to the occluded vein shortly after onset ( $<30$ days) were excluded. There were no language restrictions.

\section{Outcomes}

The primary outcome was visual acuity measured as mean change in best-corrected visual acuity (BCVA) or as proportion of patients improving by 15 Early Treatment for Diabetic Retinopathy Study (ETDRS) letters or more. Secondary outcomes included mean change in macular thickness using optical coherence tomography (OCT), quality of life and adverse events.

\section{Screening and data extraction}

Search results were screened independently by two authors (CC, JAF and ST). Differences were resolved through discussion or by consulting a third author (JAF). Data were extracted by one author (CC and DS) and checked by a second (ST, CC). Data extraction included inclusion/exclusion criteria, baseline demographics, mean change in BCVA, proportion of patients with 15 letters improvement, central retinal thickness (CRT) and adverse events. Risk of bias was assessed by two reviewers using the Cochrane risk of bias tool. ${ }^{16}$

Meta-analysis was not possible because of a lack of comparable studies. 


\section{RESULTS}

\section{Search results}

The study flow is shown in figure 1 . The electronic searches yielded 518 records. A total of 475 were eliminated based on information in the titles and abstract. The full text of the remaining 43 records was checked, and a further eight were eliminated. Reasons for exclusion included the trial being a commentary rather than an RCT, the study having no relevant comparison group (dose ranging only), the participants did not have macular oedema secondary to CRVO, or the interventions being ineligible (non-pharmacological). The remaining 35 records (including conference abstracts) reported on eight RCTs of six different pharmacological agents, and these were included in the analysis. The Geneva study $(2010)^{11} 17 \quad 18$ technically consists of two RCTs, but as these were analysed and reported together, it was counted as one RCT in this analysis.

We also identified three relevant ongoing trials, one investigating minocycline (http://clinicaltrials.gov/ct2/ show/study/NCT01468844), one investigating a combination of bevacizumab and triamcinolone (http:// clinicaltrials.gov/show/NCT00566761) and one investigating ranibizumab (http://clinicaltrials.gov/show/ NCT01123564).

\section{Study characteristics}

Detailed study characteristics of the included studies are shown in online supplementary table S1.

\section{Study design}

Of the eight included RCTs, six were described as double-blinded and seven were sham-controlled. All but one were multicentre. Only one was not funded by industry. Four trials were international trials, two came from the USA, and one each from Austria and Sweden. Six of the trials measured primary endpoints at around 6 months (24-30 weeks), whereas two measured primary endpoints at 12 months. Five studies reported follow-up data for up to 12 months, and two reported data for follow-up periods of up to 2 years.

\section{Participants}

The trials randomised a total of 1714 eyes ( 1 eye/ person). The number of eyes per study ranged between 60 and 437. Follow-up at the primary endpoint ranged from $77 \%$ to $98 \%$ (generally over $90 \%$ in the intervention groups). The participants had a mean age of between 59 and 70.5 years, and between $36 \%$ and $49 \%$ were female. Only two studies reported mean duration of macular oedema (4.3 and 4.9 months). Five studies reported mean time since CRVO diagnosis (range 2.4-2.9 months). Mean baseline BCVA was between 44 and 52.5 ETDRS letters, baseline CRT was between 569 and $721 \mu \mathrm{m}$. In most trials, the focus was on macular oedema secondary to CRVO only, but in the Geneva trial macular oedema secondary to BRVO and CRVO was included and only limited data were available on the CRVO-only group.

\section{Interventions}

The Geneva trial (2010 ff.) $)^{11} 1718$ compared a $0.35 \mathrm{mg}$ $(\mathrm{n}=136)$ and a $0.7 \mathrm{mg}$ dexamethasone $(\mathrm{n}=154)$ intravitreal implant with sham treatment $(n=147)$. After the initial 6-month study period, patients could enter a 6-month open label extension, where they received a $0.7 \mathrm{mg}$ dexamethasone intravitreal implant.

The Standard Care versus Corticosteroid for Retinal Vein Occlusion (SCORE) trial (2009 ff. $)^{19-32}$ compared intravitreal injections of 1 or $4 \mathrm{mg}$ of triamcinolone ( $\sim 2$ injections over 12 months, $\mathrm{n}=92$ and 91 for 1 and $4 \mathrm{mg}$, respectively) with an observation group $(\mathrm{n}=88)$. Two forms of triamcinolone have been used in trial; the SCORE trial used Trivaris, rather than Kenalog. Trivaris is no longer available because its manufacturer has promoted an alternative steroid (dexamethasone). The Radial Optic Neurotomy for Central Vein Occlusion (ROVO) trial (2013) ${ }^{33}$ compared a single intravitreal injection of $4 \mathrm{mg}$ of triamcinolone (over 12 months, $\mathrm{n}=25)$ with radial optic neurotomy $(\mathrm{n}=38)$ or sham injection $(n=20)$.

In the Carvedilol Prospective Randomised Cumulative Survival (COPERNICUS) trial (2012) ${ }^{34} 35$ intravitreal injections of $2 \mathrm{mg}$ of aflibercept $(\mathrm{n}=114)$ were given every 4 weeks for over 24 weeks to the intervention group and the comparison group received a sham injection $(n=75)$. During weeks 24-52, patients in both groups received aflibercept if they met protocol-specified retreatment criteria and received a sham injection if retreatment was not indicated (3.9 SE 0.3 injections in the sham group and 2.7 SE 0.2 injections in the aflibercept group); after the first year, patients continued in an 1-year extension phase with as needed dosing. In the GALILEO trial $(2012)^{36} 37$ intervention patients also received intravitreal injections of $2 \mathrm{mg}$ of aflibercept $(n=103)$ every 4 over 24 weeks, while the comparison group was given sham injections $(n=71)$. During weeks 24-52, patients remained in their original treatment groups but received their allocated treatment as needed; beginning from weeks 52 to 76 , both groups received the study drug every 8 weeks.

In a trial by Wroblewski and colleagues, ${ }^{38-44}$ patients received 0.3 or $1 \mathrm{mg}$ intravitreal injections of pegaptanib sodium every 6 for 24 weeks ( $\mathrm{n}=33$ and 33), compared with a sham injection group $(\mathrm{n}=32)$. Patients were followed up to 52 weeks.

The Central Retinal Vein Occlusion (CRUISE) trial (2010 ff.) $)^{104546}$ compared monthly injections of 0.3 or $0.5 \mathrm{mg}$ of ranibizumab $(\mathrm{n}=132$ and 130) over 6 months with sham injection $(n=130)$. During months $6-12$, all patients could receive intraocular ranibizumab (previously assigned dose or $0.5 \mathrm{mg}$ for the sham group) if they met prespecified functional and anatomic criteria; after 12 months' follow-up patients could continue in the Health Outcomes and Reduced Incidence with 
Figure 1 PRISMA statement.

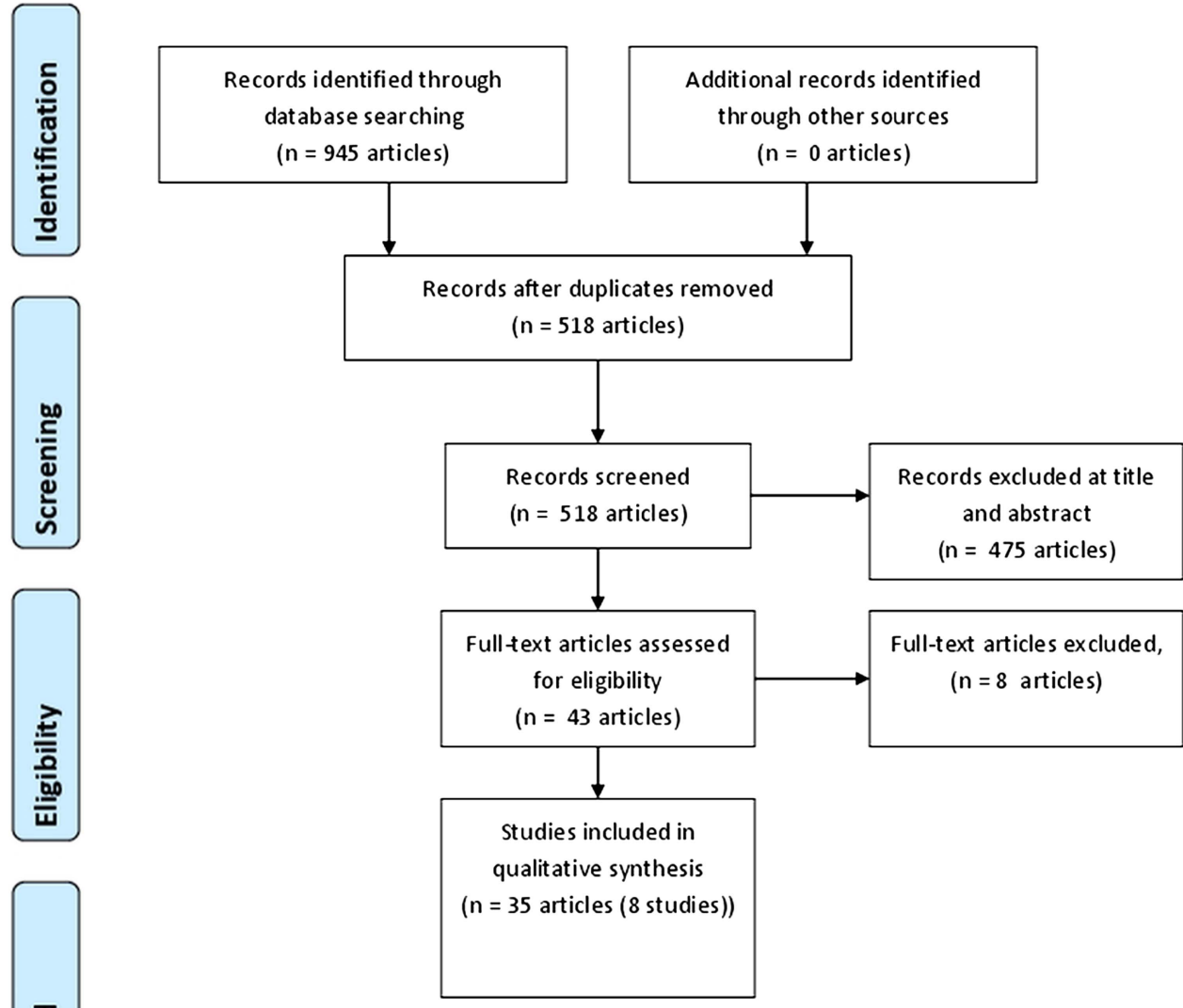

Zoledronic Acid Once Yearly (HORIZON) trial for another 12 months, where they were eligible to receive intravitreal injections of $0.5 \mathrm{mg}$ ranibizumab if they fulfilled prespecified criteria.

Epstein et $a l^{47-49}$ conducted an RCT in which they compared patients receiving four intravitreal injections of $1.25 \mathrm{mg}$ of bevacizumab ( $\mathrm{n}=30)$ over 6 months with patients receiving sham injections $(n=30)$. From 6 to 12 months, all patients received intravitreal bevacizumab injections every 6 week.

\section{Outcomes}

The primary endpoint of all but one study was the proportion with a gain of 15 or more ETDRS letters. The primary endpoint of the remaining study was mean change in BCVA. Studies also reported gains or losses of ETDRS letters at various cut-off points, absolute BCVA, CRT and safety parameters. The COPERNICUS, the GALILEO and the CRUISE studies also measured visionrelated quality of life (National Eve Institute Visual Functioning Questionnaire, NEI-VFQ). ${ }^{10} \quad 34-37 \quad 45 \quad 46$ EQ5D was also used in GALILEO.

\section{Ongoing studies}

Ongoing trials are shown in online supplementary table S4, the first (clinicaltrials.gov NCT01468844) is a 24-month double-blind RCT from the USA. It set out to test the safety and effectiveness of minocycline as a treatment for CRVO in around 20 patients with macular oedema secondary to CRVO. Both groups received monthly intravitreal bevacizumab injections over 3 months (and afterwards as needed), and the intervention group also received $100 \mathrm{mg}$ oral minocycline twice daily over 24 months. The second trial (clinicaltrials.gov NCT00566761) is an open-label RCT from Mexico in only around 10 patients assessing whether combined treatment with bevacizumab and triamcinolone is more effective than bevacizumab alone. The combination group received $2.5 \mathrm{mg}$ of bevacizumab plus $4 \mathrm{mg}$ of triamcinolone as a first dose and then two doses of bevacizumab alone at monthly intervals, while the monotherapy group received three monthly doses of $2.5 \mathrm{mg}$ bevacizumab alone. Follow-up will be 12 months. A third RCT from Hungary compares monthly injections of ranibizumab for 3 months (and as needed thereafter) with Argon laser treatment in around 40 patients with macular oedema secondary to CRVO. Follow-up will also be 12 months. The primary endpoint in all studies is BCVA over 12 months.

\section{Risk of bias}

Details of risk of bias assessment are shown in online supplementary table S3.

Most studies (except GALILEO (2012) and Epstein 2012) $363747-49$ adequately described the generation of 
the allocation sequence, but only half the studies gave enough details to confirm adequate allocation concealment. Most studies (unclear in the ROVO 2013 study) ${ }^{33}$ used at least partial masking, and most studies appeared to have had masking of outcome assessment. Intentionto-treat analysis was used in all studies. Where reported separately for comparison groups, losses to follow-up tended to be slightly higher for the control groups than the interventions groups $(79-88.5 \%$ follow-up in the control groups and $90-98 \%$ in the intervention groups). All studies appeared to have been free of selective reporting. Most studies included a power analysis (not reported for the CRUISE study ${ }^{104546}$ but in two cases (the SCORE and the ROVO studies) ${ }^{19-33}$ the numbers randomised were considerably below the numbers indicated in the power calculations. As far as reported, there were no significant differences between comparison groups in baseline characteristics.

\section{Clinical effectiveness}

Detailed study results can be found in online supplementary table S2.

\section{Visual acuity}

Figure 2 shows the primary endpoint in most studies, which was the proportion of participants with a gain of 15 or more ETDRS letters. As there were no significant differences in visual acuity results between groups using different dosages of the given pharmacological treatment, intervention groups were combined for the sake of the plot.

In the Geneva trial (2010 ff.) ${ }^{11} 17 \quad 18$ treatment of macular oedema secondary to CRVO with a $0.7 \mathrm{mg}$ intravitreal dexamethasone implant resulted in a 0.1 letter gain in BCVA compared to a loss of 1.8 in the sham group $(\mathrm{p}<0.001)$. The difference persisted in the extension period where all patients received the $0.7 \mathrm{mg}$ dexamethasone implant. However, there was no significant difference in the proportion of patients gaining or losing 15 letters at either 6 or 12 months $(0.35$ or $0.7 \mathrm{mg}$ dexamethasone). This may reflect the timing of peak effect at 90 days with dexamethasone.

In the SCORE trial (2009 ff.), ${ }^{19-32}$ patients in the triamcinolone groups lost significantly fewer ETDRS letters (triamcinolone $1 \mathrm{mg} 1.2$ letters loss, $4 \mathrm{mg} 1.2$ letters loss and observation 12.1 letters loss) over both 12 and 24 months than patients in the observation group. The proportion of patients gaining 15 letters or more was also significantly larger in the intervention groups at 12 and 24 months $(25.6 \%$ compared with $6.8 \%$ and $31 \%$ compared with $9 \%$, respectively). The proportion of patients receiving triamcinolone and losing 15 letters or more was smaller $(25.6 \%)$ than in the observation group (43.8\%), but this difference was not statistically significant $(\mathrm{p}=0.06)$.

There was some overall improvement in BCVA in both intervention groups at 12 months in the ROVO trial $\left(2013^{33}\right.$; triamcinolone $20 \%$, radial optic neurotomy $47 \%$ and sham $10 \%$ ); however, it was unclear whether there were any statistically significant differences between the $4 \mathrm{mg}$ triamcinolone, the radial optic neurotomy or the sham group. However, there were significantly more patients with an improvement of more than or equal to 15 letters in the neurotomy group than in the sham group ( $47 \%$ vs $10 \%$ ), but no significant difference to sham after one dose of triamcinolone.

In the COPERNICUS (2012) ${ }^{34} 35$ and GALILEO $(2012)^{36} 37$ trials, patients in the aflibercept group had a significant improvement in BCVA at 6 months of 18 and 17.3 letters (compared with 4 letters loss and 3.3 letter gain in sham groups, respectively) and this was maintained at 12 months and was significantly greater than the improvements in the sham groups. This was paralleled by a significantly greater proportion of patients
Figure 2 Study results for the primary outcome $(\geq 15$ Early Treatment for Diabetic Retinopathy Study letter gain).

\begin{tabular}{|c|c|c|c|c|c|c|c|c|}
\hline \multirow{3}{*}{$\frac{\text { Study or Subgroup }}{1.1 .1-6 \text { months }}$} & \multicolumn{2}{|c|}{ Experimental } & \multicolumn{2}{|c|}{ Control } & \multirow{2}{*}{$\begin{array}{c}\text { Risk Ratio } \\
\mathrm{M} \cdot \mathrm{H}, \text { Random, } 95 \% \mathrm{Cl}\end{array}$} & \multirow{2}{*}{\multicolumn{3}{|c|}{$\begin{array}{c}\text { Risk Ratio } \\
\mathrm{M} \cdot \mathrm{H}, \text { Random, } 95 \% \mathrm{Cl}\end{array}$}} \\
\hline & \multirow[t]{2}{*}{ Events } & \multirow[t]{2}{*}{ Total } & \multirow[t]{2}{*}{ Events } & \multirow[t]{2}{*}{ Total } & & & & \\
\hline & & & & & & & & \\
\hline Aflibercept COPERNICUS & 64 & 114 & 9 & 73 & $4.55[2.42,8.57]$ & & & \\
\hline Aflibercept GALILEO & 62 & 103 & 15 & 68 & $2.73[1.70,4.38]$ & & & \\
\hline Bevacizumab Epstein & 18 & 30 & 6 & 30 & $3.00[1.38,6.50]$ & & & \\
\hline Dexamethasone GENEVA & 51 & 290 & 18 & 147 & $1.44[0.87,2.37]$ & & & \\
\hline Pegaptanib Wroblewski & 25 & 66 & 9 & 32 & $1.35[0.71,2.54]$ & & & \\
\hline Ranbizumab CRUISE & 123 & 262 & 22 & 130 & $2.77[1.86,4.15]$ & & & \\
\hline 1.1.2 - 12 months & & & & & & & & \\
\hline Aflbercept COPERNICUS & 63 & 114 & 22 & 73 & $1.83[1.25,2.70]$ & & 千 & \\
\hline Aflbercept GALILEO & 62 & 103 & 22 & 68 & $1.86[1.28,2.71]$ & & 7 & \\
\hline Bevacizumab Epstein & 18 & 30 & 10 & 30 & $1.80[1.00,3.23]$ & & + & \\
\hline Ranbizumab CRUISE & 128 & 262 & 43 & 130 & $1.48[1.12,1.94]$ & & 十 & \\
\hline Triamcinolone SCORE & 43 & 165 & 5 & 73 & $3.80[1.57,9.21]$ & & & \\
\hline 1.1.3 - 24 months & & & & & & & & \\
\hline Ranbizumab CRUISE & 86 & 206 & 38 & 98 & $1.08[0.80,1.45]$ & & & \\
\hline Triamcinolone SCORE & 30 & 105 & 4 & 46 & $3.29[1.23,8.79]$ & & & \\
\hline & & & & & & $1 \quad 1$ & + & 1 \\
\hline & & & & & & $\begin{array}{llll}0.1 & 0.2 & 0.5 & 1\end{array}$ & 125 & 10 \\
\hline & & & & & & Favours control & Favours exp & erimenta \\
\hline
\end{tabular}


(56.1\% compared with $12.3 \%$ and $60.2 \%$ compared with $22.1 \%$, respectively) gaining 15 letters or more. Patients treated sooner after diagnosis (less than vs more than 2 months) seemed to benefit more (in terms of proportion of patients with 15 letters or more gain) in both trials.

The increase in mean change in BCVA with $0.3 \mathrm{mg}$ pegaptanib compared with sham did not reach significance at 30 weeks in the trial by Wroblewski and colleagues, ${ }^{38-44}$ but there was a greater increase in BCVA with $1 \mathrm{mg}$ pegaptanib compared with sham (9.9 letter gain compare with 3.2 letter loss). These differences were not statistically significant at 52 weeks. There was no significant difference between any of the groups in the proportion of patients gaining 15 letters or more at 30 weeks, but significantly fewer patients in both dosage groups lost 15 letters or more than in the sham group $(6 \%$ compared with $31 \%$ ).

In the CRUISE trial (2010 ff.) $)^{104546}$ mean change in BCVA was significantly increased in the ranibizumab groups (no difference between doses) compared with the sham group at 6 and 12 months (12 letters gained in the $0.5 \mathrm{mg}$ group compared with 7.6 in the sham group). After the 1 year extension with ranibizumab as needed in all groups, there was no difference between the doses of ranibizumab at 24 months. The pattern was similar for the proportion of patients gaining 15 letters or more.

In the trial by Epstein $e t a l,{ }^{47-49}$ treatment with intravitreal bevacizumab, compared with sham treatment significantly increased mean change in BCVA (14.1 letters gain compared with 2 letters lost) and the proportion of patients gaining 15 letters or more (60\% compared to $20 \%)$ at 24 weeks. This difference was maintained in the extension period, even though both groups had been receiving bevacizumab. Younger patients ( $<70$ years) tended to have better visual outcomes than older patients ( $>70$ years).

\section{Central retinal thickness}

In the Geneva trial (2010 ff.) ${ }^{111718}$ no significant difference was found in the reduction of CRT after 6 months of treatment in patients with macular oedema secondary to CRVO with the $0.7 \mathrm{mg}$ intravitreal dexamethasone implant (no data given for the $0.35 \mathrm{mg}$ implant) compared with sham.

In the SCORE trial (2009 ff.), ${ }^{19-32}$ CRT decreased in all study groups, but there was no significant difference between groups at either 12 or 24 months. Similarly, there was no clear difference in the proportion of patients achieving a CRT of less than $250 \mu \mathrm{m}$. CRT decreased in all comparison groups in the ROVO trial $(2013)^{33}$ but there was no significant difference between groups.

In the COPERNICUS trial (2012) ${ }^{34} 35$ and in the GALILEO trial (2012) ${ }^{36} 37$ there was a significantly greater reduction in CRT at 6 months in the aflibercept group than in the control group. However, the significant difference was maintained in the longer term only in the GALILEO trial, where patients continued their assigned treatment up to 12 months. In the COPERNICUS trial, patients in the sham group also received aflibercept in the extension period, which caused a similar decrease in CRT as in the original intervention group.

After 30 weeks of treatment with pegaptanib (Wroblewski and colleagues), ${ }^{38-44}$ differences in decrease of CRT versus sham did not reach significance, but at 52 weeks, the decrease in CRT was significantly greater in both the $0.3 \mathrm{mg}$ and the $1 \mathrm{mg}$ pegaptanib groups compared with sham.

After treatment with ranibizumab in the CRUISE trial (2010 ff.) ${ }^{10} 45{ }^{46}$ a significant reduction in CRT was observed and significantly more patients achieved a CRT of $250 \mu \mathrm{m}$ or less in the intervention groups (no difference between doses) than in the sham group at 6 months. This difference did not persist at 12 and 24 months because all groups received ranibizumab as needed.

In the trial by Epstein $e t a l,{ }^{47-49}$ treatment with intravitreal bevacizumab significantly decreased CRT and the proportion of patients with no residual oedema (CRT $<300 \mu \mathrm{m})$ at 24 weeks, compared with sham treatment. When both groups received bevacizumab in the extension period, similar decreases in CRT and increases in the proportion of patients with no residual oedema were seen.

\section{Vision-related quality of life}

Vision-related quality of life (NEI-VFQ25) was significantly higher in the aflibercept group, compared with sham injection, at 6 months in the COPERNICUS trial $(+7.2 \text { compared with }+0.8)^{34} 35$ and the GALILEO trial $(+7.5$ compared with +3.5$) .{ }^{36} 37$ In the COPERNICUS trial, patients in the sham group who received aflibercept in the extension period had a similar increase in vision-related quality of life as patients in the original intervention group by 12 months.

In the CRUISE trial (2010 ff.) ${ }^{104546}$ vision-related quality of life (NEI-VFQ) was similarly increased in both ranibizumab groups and statistically significantly more than in the sham group at 6 months $(+6.2$ compared with +2.8$)$. At 12 months, with all groups receiving ranibizumab as needed, the increases were similar in all three groups.

\section{Adverse events}

The $0.7 \mathrm{mg}$ dexamethasone intravitreal implant caused significantly more increased intraocular pressure (IOP) than sham treatment $(30.1 \%$ vs $1.4 \%$ in the control group) in patients with CRVO in the Geneva trial (2010 $\mathrm{ff}^{11}{ }^{17}{ }^{18}$; not reported for $0.35 \mathrm{mg}$ ). The incidence of cataract was also slightly higher in the dexamethasone group but numbers were small because of the short duration. There were no other differences in adverse events between groups. 
In the triamcinolone group (especially $4 \mathrm{mg}$, SCORE trial 2009 ff.) ${ }^{19-32}$ there was a higher increase in IOP, lens opacity onset or progression (at 12 months) and cataract surgery (12-24 months) than in the control group. There were no other differences in adverse events between groups. A similar tendency was seen in the ROVO trial (2013). ${ }^{33}$

Aflibercept did not appear to increase the incidence of ocular or non-ocular adverse events compared with sham in both the COPERNICUS trial (2012) ${ }^{34} 35$ and the GALILEO trial (2012). ${ }^{36} 37$

In the trial by Wroblewski and colleagues, ${ }^{38-44}$ adverse events in response to pegaptanib were not reported in detail, but there do not appear to have been any serious ocular or systemic adverse events.

After treatment with ranibizumab in the CRUISE trial (2010 ff.) ${ }^{104546}$ there were no consistent differences in ocular or systemic adverse events between the intervention groups. None of the ocular adverse events appeared to have increased substantially after all patients received ranibizumab up to 24 months.

Epstein et $a t^{77-49}$ did not report adverse events in response to bevacizumab in detail, but the treatment appears not to have caused any serious ocular adverse events over 48 weeks.

\section{DISCUSSION}

\section{Statement of principal findings}

Evidence from good quality RCTs shows that intravitreal steroids and anti-VEGF therapies increase the proportion of patients whose vision improves by 15 or more letters in patients with macular oedema secondary to CRVO. The most effective drugs result in over $60 \%$ of patients gaining 15 letters compared with only about $20 \%$ of the control groups. The RCT evidence shows only short-term effectiveness of ranibizumab, bevacizumab, aflibercept and triamcinolone. Results from trials of dexamethasone and pegaptanib were mixed. Long-term evidence is awaited.

\section{Strengths and limitations}

A robust systematic review methodology was used. A broad search strategy was implemented, which included not restricting the search strategy with drug terms. Grey literature was searched by screening meeting abstracts from relevant conferences. There were no language restrictions. Two reviewers screened titles and abstracts and conducted data extraction and risk of bias assessment. Risk of bias was assessed using the Cochrane Risk of Bias Tool and was generally judged to be low or unclear. Only studies with 1-year follow-up were included to exclude studies with very short follow-up RCTs were identified for all the new ophthalmological drugs, except for the steroid, fluocinolone.

The main limitation is the short duration of follow-up. The primary outcome for most trials was measured at 6 months, with an extension phase of up to 12 months.
Hence, it is not known whether the benefit of these treatments will be maintained long term. Furthermore, potential side effects of these treatments may not be captured in these studies as a result of their short follow-up. Patients and clinicians would like sustained, life-long improvement in visual acuity, but of all included studies only one of them had a follow-up of over 24 months.

The sample size of some studies was small. For example, the evidence for pegaptanib and bevacizumab comes from studies with around 30 participants/arm which substantially increases the risk of a type II error. Only three trials included quality of life data, arguably one of the most important outcomes.

The proportion of participants and severity of ischaemia within the trials was not clear. While ischaemia is not mentioned in the inclusion/exclusion criteria of most studies, these participants were unlikely included in these studies, especially if the diagnosis of ischaemic CRVO is based on strict criteria. Furthermore, patients were entered into the trials relatively soon after diagnosis (mean 4.3-4.9 months) and it is not clear if the effects would be similar in patients who present with longstanding disease.

Another limitation was that patients were not asked at the trials, what treatment they thought they had received, which would have provided data on the success of masking of allocation.

In the case of dexamethasone, the results at 6 months were not as good as at 90 days, because of the duration of action. Earlier retreatment, at say 120 days, would have improved results, but many clinicians might be reluctant to repeat injections of dexamethasone implant often because of the large needle size and risk of adverse effects.

\section{Adverse events}

Results from the included studies clearly demonstrate that steroids (triamcinolone and dexamethasone) are associated with clinically meaningful increases in IOP and cataract progression. Anti-VEGF therapy ocular adverse events reported in the trials were similar in both placebo and intervention arms.

There is limited evidence of the safety of these drugs specifically in CRVO, but it would not be unreasonable to look to trials in neovascular AMD and diabetic macular oedema (DMO) for safety data, where there is more experience. The comparison of AMD treatment trial, which compared bevacizumab with ranibizumab in AMD, suggested that there was a higher incidence (relative risk (RR) 1.29 ; $95 \%$ CI 1.01 to 1.66 ) of serious systematic adverse events (primarily hospitalisations) in the bevacizumab arm. ${ }^{50}$ Some have raised concerns about arterial thromboembolic events with bevacizumab, but none of these has been demonstrated in the published literature. ${ }^{51-54}$ Micieli et $a \bar{l}^{55}$ undertook a systematic review of the adverse events associated with bevacizumab. Twenty-two studies were reviewed, representing 12699 participants. ${ }^{55}$ Adverse events in patients treated 
with bevacizumab were cerebrovascular events $(0.21 \%)$, myocardial infarction $(0.19 \%)$ and increased blood pressure $(0.46 \%)$. Most of these represent the background burden of disease in patients with advanced eye disease. The proportion of these directly attributable to bevacizumab is likely to be very small. Campbell $e t a \tilde{l}^{1}$ undertook a nested case-control study of over 7000 cases and 37000 controls. Ranibizumab and bevacizumab injection was the exposure and cardiovascular events were the outcome. The authors found that ranibizumab and bevacizumab were not associated with increased cardiovascular events.

Increased IOP has been associated with ranibizumab, bevacizumab and pegaptanib. Sustained increased in IOP has estimated to be $5.5-6 \%$ with these drugs. ${ }^{56} 57$

Robust evidence on the long-term safety of aflibercept is awaited.

\section{What do these results mean?}

Until very recently, patients with macular oedema as a result of CRVO could only be offered visual rehabilitation and visual aids in an attempt to help them to deal better with their reduced vision and its implications in their daily activities and quality of life. Their future is brighter now as new options to treat macular oedema have become available. Triamcinolone is likely to be a cost-effective treatment at least in selected groups of patients, such as pseudophakic individuals or those with pre-existing cataracts that may require cataract surgery in the near future. The lack of a commercially available licensed product for intraocular administration may restrict its use in clinical practice.

Some anti-VEGF therapies, including bevacizumab, ranibizumab and aflibercept, have also been shown to be effective in short-term studies for the treatment of patients with macular oedema and CRVO. Bevacizumab has the advantage of having a low cost, with an apparently similar effect to other anti-VEGF therapies ${ }^{50} 5859$ but there is some reluctance to use it as it is not licensed for use in the eye. This has been seen in other eye conditions, such as AMD and DMO. Aflibercept, requiring potentially fewer injections than other anti-VEGF agents, could represent an advantage to patients and may relieve pressure on ophthalmology clinics. Healthcare systems will need to evaluate the cost-effectiveness of these new treatments and support affordable ones. The NICE is currently appraising aflibercept. Policy makers are left in a difficult position because of bevacizumab. It is cheaper than all other drugs ${ }^{60}$ and appears to be as effective, but is unlicensed and unlike ranibizumab and aflibercept does not have evidence from large, wellfunded RCTs in CRVO. The use of bevacizumab would result in considerable savings for the NHS.

It is important to note that the evidence of benefit of these new therapies is likely to only apply to patients with non-ischaemic CRVO. Although some patients with ischaemic CRVO were included, these individuals are likely to have mild ischaemic CRVO. Thus, for patients with established ischaemic CRVO, there are no proven treatments available and further research into this area is very much needed.

\section{What is the context of these results}

Earlier systematic reviews identified limited evidence on the clinical effectiveness of treatments. A review by Braithwaite $e t a l^{61}$ (search date August 2010) on anti-VEGF agents identified one RCT ${ }^{104546}$ comparing two doses of ranibizumab and one $\mathrm{RCT}^{38-44}$ comparing two doses of pegaptanib sodium versus placebo or no treatment. In both RCTs, the higher dose of the anti-VEGF significantly improved BCVA compared with sham injection in the short term ( $\sim 6$ months), but the effects in the longer term were unclear. Braithwaite and colleagues concluded that data from the two RCTs could not be synthesised because ranibizumab and pegaptanib sodium might not be directly comparable. Subsequent RCTs identified in this review also suggest benefit in ocular outcomes in macular oedema secondary to non-ischaemic CRVO for the anti-VEGFs bevacizumab and aflibercept. ${ }^{34-37} 47-49$

Gewaily and Greenberg ${ }^{62}$ reviewed the literature on intravitreal corticosteroids (search date November 2008) versus observation in macular oedema secondary to CRVO and identified no relevant RCTs. Results from two observational studies suggested that triamcinolone acetonide might be beneficial in the treatment of macular oedema secondary to non-ischaemic CRVO. However, as the authors of the review caution because conclusions are primarily drawn from small case series and case reports with short follow-up. Results from the SCORE 2009 RCT corroborate the observational studies. ${ }^{19-32}$ The effects of triamcinolone acetonide in people with non-ischaemic CRVO without associated macular oedema are less clear. Data from four observational studies led Gewaily and Greenberg to conclude that intravitreal corticosteroids are associated with transient anatomical and functional improvements.

Immediate treatment aimed at relieving the blocked vein and surgical interventions were out with the remit of this review. Antithrombotics, such as low-molecular weight heparin (LMWH), and fibrinolytics have also been found to benefit visual acuity in retinal vein occlusion with no associated macular oedema. Two systematic reviews ${ }^{63}{ }^{64}$ identifying the same three RCTs in recent onset ( $\leq 30$ days) BRVO or CRVO found that LMWH improved visual acuity compared with aspirin and that the associated benefit was larger in CRVO; only one of the three RCTs included people solely with CRVO. One review $^{64}$ also included one RCT comparing ticlopidine with placebo and two RCTs assessing intravenous fibrinolytic therapy followed by warfarin or aspirin with either haemodilution or no treatment. The authors of the reviews conclude that no definitive recommendations can be made on clinical effectiveness of LMWH in CRVO given the limited evidence available.

Radial optic neurotomy involves the performance of a radial cut using a microvitreoretinal blade through the 
lamina cribrosa, scleral ring and adjacent sclera at a selected point in the optic nerve head with the goal of 'decompressing' the scleral outlet (space confined by the scleral ring and containing the lamina cribrosa, the central retinal artery, central retinal vein and the optic nerve). The ROVO trial found radial optic neurotomy to be more effective than sham.

While this review was being considered for publication, another was published, with differences in scope (BRVO and CRVO) and inclusions (this review is more up-to-date). ${ }^{65}$ The reviewers found that aflibercept and bevacizumab resulted in greatest gain, followed by ranibizumab and triamcinolone. The overall conclusions in both reviews were similar.

\section{Further research}

Large adequately powered RCTs comparing ranibizumab, bevacizumab, aflibercept and triamcinolone are needed. Part of the problem is that the US Food and Drug Administration requires pharmaceutical companies to present data establishing a drug's safety and effectiveness. While this does not specifically require a placebocontrolled trial, it is the most efficient study design for demonstrating effectiveness and safety. Clinicians and researchers are left with placebo-controlled trials demonstrating effectiveness for individual drugs, but a lack of evidence to help them decide which is best for their patients.

Given the cost of these treatments and the burden of repeated injections to patients and healthcare systems, research aiming to predict 'responders' would be useful as at present this is performed by therapeutic trials. Treatments could then be targeted to patients likely to benefit. Research is also needed on the frequency and sequences of drugs. As other pathogenic pathways besides inflammation and VEGF-mediated pathways may be implicated in the development of macular oedema in patients with CRVO, these should be investigated in an attempt to develop new therapeutic strategies for this condition. Research is also needed into optimum timing of treatment after CRVO. The cost-effectiveness of diagnostic technologies for determining when retreatment is necessary should be examined.

We also need better treatments since a significant proportion of patients do not improve with all of these drugs.

Future RCTs should include longer term outcomes, as functional results observed at 6 months or even 1 year may not necessarily be representative of what is likely to be achieved longer term and, furthermore, potential side effects of treatments, such as retinal atrophy after repeated injections of anti-VEGFs, may not be captured in short-term studies.

\section{CONCLUSIONS}

Bevacizumab, ranibizumab, aflibercept and triamcinolone appear to be effective in improving the number of patients who gain 15 letters or more in CRVO. There are mixed results for dexamethasone and pegaptanib. Steroids were associated with cataract progression and increased IOP. Long-term data on effectiveness and safety are needed. Head-to-head trials and research to identify 'responders' is needed to help clinicians make the right choices for their patients.

\section{Author affiliations}

${ }^{1}$ Faculty of Medicine and Health Sciences, University of East Anglia, Norwich, UK

${ }^{2}$ Warwick Evidence, University of Warwick, Coventry, UK

${ }^{3}$ Centre for Vision and Vascular Science, Queen's University, Belfast, UK

${ }^{4}$ BMJ Technology Assessment Group, London, UK

${ }^{5}$ Division of Health Sciences, Medical School, University of Warwick, Coventry, UK

Contributors NW devised the idea for the review. JAF wrote the protocol and all authors contributed to the design of the protocol. RC undertook the literature searches. JAF, CC and ST screened titles and abstracts. CC, ST and DS extracted the data. All authors contributed to the interpretation of the results. JAF, NL, RC, CC and SB contributed to the first draft of the article. All authors reviewed and commented on the final manuscript.

Competing interests None.

Provenance and peer review Not commissioned; externally peer reviewed. Data sharing statement No additional data are available.

Open Access This is an Open Access article distributed in accordance with the Creative Commons Attribution Non Commercial (CC BY-NC 3.0) license, which permits others to distribute, remix, adapt, build upon this work noncommercially, and license their derivative works on different terms, provided the original work is properly cited and the use is non-commercial. See: http:// creativecommons.org/licenses/by-nc/3.0/

\section{REFERENCES}

1. Deramo VA, Cox TA, Syed AB, et al. Vision-related quality of life in people with central retinal vein occlusion using the 25 -item National Eye Institute Visual Function Questionnaire. Arch Ophthalmol 2003;121:1297-302.

2. Mclntosh RL, Rogers SL, Lim L, et al. Natural history of central retinal vein occlusion: an evidence-based systematic review. Ophthalmology 2010;117:1113-23.

3. Mitchell P, Smith W, Chang A. Prevalence and associations of retinal vein occlusion in Australia. The Blue Mountains Eye Study. Arch Ophthalmol 1996;114:1243-7.

4. Rogers S, McIntosh RL, Cheung N, et al. The prevalence of retinal vein occlusion: pooled data from population studies from the United States, Europe, Asia, and Australia. Ophthalmology 2010;117:313-19.

5. The Royal College of Ophthalmology. Interim guidelines for management of retinal vein occlusion. http://www.rcophth.ac.uk/core/ core_picker/download.asp?id=728\&filetitle=Interim+Guidelines+for +Management+of+Retinal+Vein+Occlusion+2010 [2010 [cited 7 Sep 2013].

6. Hayreh SS, Podhajsky PA, Zimmerman MB. Natural history of visual outcome in central retinal vein occlusion. Ophthalmology 2011;118:119-33.

7. Kiire CA, Chong NV. Managing retinal vein occlusion. BMJ 2012;344:e499.

8. The Branch Vein Occlusion Study Group. Argon laser photocoagulation for macular edema in branch vein occlusion. Am J Ophthalmol 1984;98:271-82.

9. The Central Vein Occlusion Study Group. Evaluation of grid pattern photocoagulation for macular edema in central vein occlusion. Ophthalmology 1995;102:1425-33.

10. Brown DM, Campochiaro PA, Singh RP, et al. Ranibizumab for macular edema following central retinal vein occlusion: six-month primary end point results of a phase III study. Ophthalmology 2010;117:1124-33.

11. Haller JA, Bandello F, Belfort R Jr, et al Randomized, shamcontrolled trial of dexamethasone intravitreal implant in patients with macular edema due to retinal vein occlusion. Ophthalmology 2010;117:1134-46. 
12. Cunningham MA, Edelman JL, Kaushal S. Intravitreal steroids for macular edema: the past, the present, and the future. Surv Ophthalmol 2008;53:139-49.

13. Miller JW, Le CJ, Strauss EC, et al. Vascular endothelial growth factor A in intraocular vascular disease. Ophthalmology 2013:120:106-14.

14. Ford JA, Lois N, Royle $\mathrm{P}$, et al. Current treatments in diabetic macular oedema: systematic review and meta-analysis. BMJ Open 2013;3.

15. Shyangdan D, Cummins E, Lois N, et al. Dexamethasone implants in the treatment of macular oedema due to retinal vein occlusion: a single technology appraisal. Aberdeen HTA Group, 2010. http:// www.nice.org.uk/nicemedia/live/13037/52883/52883.pdf

16. Higgins J, Altman D, Gøtzsche PC, et al. The Cochrane Collaboration's tool for assessing risk of bias in randomised trials. BMJ 2011;343:d5928.

17. Haller JA, Bandello F, Belfort R Jr,, et al Dexamethasone intravitreal implant in patients with macular edema related to branch or central retinal vein occlusion twelve-month study results. Ophthalmology 2011;118:2453-60.

18. Yeh WS, Haller JA, Lanzetta $P$, et al. Effect of the duration of macular edema on clinical outcomes in retinal vein occlusion treated with dexamethasone intravitreal implant. Ophthalmology 2012;119:1190-8.

19. Bhavsar AR, Ip MS, Glassman AR, DRCRnet and the SCORE Study Groups. The risk of endophthalmitis following intravitreal triamcinolone injection in the DRCRnet and SCORE clinical trials. Am J Ophthalmol 2007:144:454-6.

20. Blodi BA, Domalpally A, Scott IU, et al. Standard Care vs Corticosteroid for Retinal Vein Occlusion (SCORE) Study system for evaluation of stereoscopic color fundus photographs and fluorescein angiograms: SCORE Study Report 9. Arch Ophthalmol 2010;128:1140-5

21. Chan CK, Ip MS, VanVeldhuisen PC, et al. SCORE Study report \#11: incidences of neovascular events in eyes with retinal vein occlusion. Ophthalmology 2011;118:1364-72.

22. Ip M, Oden N, VanVeldhuisen $\mathrm{P}$, et al. The Standard Care vs. Corticosteroid for Retinal Vein Occlusion Study: design and baseline characteristics. Am Acad Ophthalmol 2008;PO285.

23. Ip MS, Scott IU, VanVeldhuisen PC, et al. A randomized trial comparing the efficacy and safety of intravitreal triamcinolone with observation to treat vision loss associated with macular edema secondary to central retinal vein occlusion: the Standard Care vs Corticosteroid for Retinal Vein Occlusion (SCORE) study report 5. Arch Ophthalmol 2009:127:1101-14.

24. Ip MS, Oden NL, Scott IU, et al. SCORE Study report 3: study design and baseline characteristics. Ophthalmology 2009;116:1770-7.

25. Myers D, Blodi B, Ip M, et al. Reading Center Evaluation of OCT Images From Patients Enrolled in the Standard Care vs. Corticosteroid for Retinal Vein Occlusion (SCORE) Study. IOVS 2006;47:ARVO E-abstract 5194

26. Oden NL, Veldhuisen PC, Scott IU, et al. Temporal Variability of OCT in Retinal Vein Occlusion Participants in the SCORE Study. IOVS 2007;48:ARVO E-Abstract 107.

27. Scott IU, VanVeldhuisen PC, Oden NL, et al. SCORE Study report 1: baseline associations between central retinal thickness and visual acuity in patients with retinal vein occlusion. Ophthalmology 2009:116:504-12.

28. Scott IU, Blodi BA, Ip MS, et al. SCORE Study Report 2: interobserver agreement between investigator and reading center classification of retinal vein occlusion type. Ophthalmology 2009;116:756-61.

29. Scott IU, Oden NL, VanVeldhuisen PC, et al. SCORE Study Report 7: incidence of intravitreal silicone oil droplets associated with staked-on vs luer cone syringe design. Am J Ophthalmol 2009;148:725-32.

30. Scott IU, VanVeldhuisen PC, Oden NL, et al. Baseline predictors of visual acuity and retinal thickness outcomes in patients with retinal vein occlusion: Standard Care Versus COrticosteroid for REtinal Vein Occlusion Study report 10. Ophthalmology 2011;118:345-52.

31. Scott IU, VanVeldhuisen PC, Oden NL, et al. Baseline characteristics and response to treatment of participants with hemiretinal compared with branch retinal or central retinal vein occlusion in the Standard Care vs COrticosteroid for REtinal Vein Occlusion (SCORE) study: SCORE Study report 14. Arch Ophthalmol 2012;130:1517-24.

32. Warren K, Blodi BA, Oden N, et al. Reading center evaluation of baseline retinal images in the Standard Care vs. Corticosteroid for Retinal Vein Occlusion (SCORE) Study. IOVS 2008;ARVO Eabstract 2136 .
33. Aggermann $\mathrm{T}$, Brunner S, Krebs I, et al. A prospective, randomised, multicenter trial for surgical treatment of central retinal vein occlusion: results of the Radial Optic Neurotomy for Central Vein Occlusion (ROVO) study group. Graefes Arch Clin Exp Ophthalmol 2013;251:1065-72.

34. Boyer D, Heier J, Brown DM, et al. Vascular endothelial growth factor Trap-Eye for macular edema secondary to central retinal vein occlusion: six-month results of the phase 3 COPERNICUS study. Ophthalmology 2012;119:1024-32.

35. Brown DM, Heier JS, Clark WL, et al. Intravitreal aflibercept injection for macular edema secondary to central retinal vein occlusion: 1 -Year Results From the Phase 3 COPERNICUS Study. Am J Ophthalmol 2013;155:429-37.

36. Gillies M. Intravitreal VEGF trap-eye in central retinal vein occlusion: results of the phase 3 copernicus and galileo studies. Clin Exp Ophthalmol 2012;40:44

37. Holz FG, Roider J, Ogura $Y$, et al. VEGF trap-Eye for macular oedema secondary to central retinal vein occlusion: 6-month results of the phase III GALILEO study. Br J Ophthalmol 2013;97:278-84.

38. Ciulla TA. Treatment of macular edema following central retinal vein occlusion with pegaptanib sodium (macugen): a one-year study. Am Acad Ophthalmol 2007;PA085

39. Csaky KG. Pegaptanib (Macugen) for Macular edema in Central Retinal Vein Occlusion: early OCT results and effect of therapy reinitiation. Am Acad Ophthalmol 2007;PO461.

40. Patel SS. Pegaptanib sodium for the treatment of macular edema following Central Retinal Vein Occlusion (CRVO): anatomical outcomes. IOVS 2007;48:ARVO E-Abstract 311.

41. Wells JA. Pegabtanib sodium for treatment of macular edema secondary to Central Retinal Vein Occlusion (CRVO). IOVS 2006:47:ARVO E-abstract 4279.

42. Wells JA. Safety and efficacy of pegaptanib sodium in treating macular edema secondary to Central Retinal Vein Occlusion. Am Acad Ophthalmol 2006;PO370.

43. Wells JA, Wroblewski JJ. Pegaptanib sodium for the treatment of macular edema following Central Retinal Vein Occlusion (CRVO): functional outcomes. IOVS 2007;48:ARVO E-Abstract 1544.

44. Wroblewski JJ, Wells JA III, Adamis AP, et al Pegaptanib sodium for macular edema secondary to central retinal vein occlusion. Arch Ophthalmol 2009;127:374-80.

45. Campochiaro PA, Brown DM, Awh CC, et al. Sustained benefits from ranibizumab for macular edema following central retinal vein occlusion: twelve-month outcomes of a phase III study. Ophthalmology 2011:118:2041-9.

46. Heier JS, Campochiaro PA, Yau L, et al. Ranibizumab for macular edema due to retinal vein occlusions: long-term follow-up in the HORIZON trial. Ophthalmology 2012;119:802-9.

47. Epstein D, Algvere P, Von WG, et al. Long-term benefit from bevacizumab for macular edema in central retinal vein occlusion: 12-month results of a prospective study. Acta Ophthalmol 2012;90:48

48. Epstein DL, Algvere PV, von Wendt G, et al. Benefit from bevacizumab for macular edema in central retinal vein occlusion: twelve-month results of a prospective, randomized study. Ophthalmology 2012;119:2587-91.

49. Epstein DL, Algvere PV, Von WG, et al. Bevacizumab for macular edema in central retinal vein occlusion: a prospective, randomized, double-masked clinical study. Ophthalmology 2012;119:1184-9.

50. Martin DF, Maguire MG, Ying GS, et al. Ranibizumab and bevacizumab for neovascular age-related macular degeneration. N Engl J Med 2011;364:1897-908.

51. Campbell RJ, Gill SS, Bronskill SE, et al. Adverse events with intravitreal injection of vascular endothelial growth factor inhibitors: nested case-control study. BMJ 2012;345:e4203.

52. Curtis LH, Hammill BG, Schulman KA, et al. Risks of mortality, myocardial infarction, bleeding, and stroke associated with therapies for age-related macular degeneration. Arch Ophthalmol 2010;128:1273-9.

53. Hwang DJ, Kim YW, Woo SJ, et al. Comparison of systemic adverse events associated with intravitreal anti-VEGF injection: ranibizumab versus bevacizumab. J Korean Med Sci 2012;27:1580-5.

54. Sharma S, Johnson D, Abouammoh M, et al. Rate of serious adverse effects in a series of bevacizumab and ranibizumab injections. Can J Ophthalmol 2012;47:275-9.

55. Micieli JA, Micieli A, Smith AF. Identifying systemic safety signals following intravitreal bevacizumab: systematic review of the literature and the Canadian Adverse Drug Reaction Database. Can J Ophthalmol 2010;45:231-8.

56. Choi DY, Ortube MC, McCannel CA, et al. Sustained elevated intraocular pressures after intravitreal injection of bevacizumab, ranibizumab, and pegaptanib. Retina 2011;31:1028-35. 
57. Good TJ, Kimura AE, Mandava N, et al. Sustained elevation of intraocular pressure after intravitreal injections of anti-VEGF agents. Br J Ophthalmol 2011;95:1111-14.

58. Chakravarthy U, Harding SP, Rogers CA, et al. Ranibizumab versus bevacizumab to treat neovascular age-related macular degeneration: one-year findings from the IVAN randomized trial. Ophthalmology 2012;119:1399-411.

59. Ford JA, Elders A, Shyangdan D, et al. The relative clinical effectiveness of ranibizumab and bevacizumab in diabetic macular oedema: an indirect comparison in a systematic review. BMJ 2012;345:e5182.

60. Stewart MW. Aflibercept (VEGF Trap-eye): the newest anti-VEGF drug. Br J Ophthalmol 2012;96:1157-8.

61. Braithwaite T, Nanji AA, Greenberg PB. Anti-vascular endothelial growth factor for macular edema secondary to central retinal vein occlusion. Cochrane Database Syst Rev 2010;(10):CD007325.

62. Gewaily D, Greenberg PB. Intravitreal steroids versus observation for macular edema secondary to central retinal vein occlusion. Cochrane Database Syst Rev 2009;(1):CD007324.

63. Lazo-Langner A, Hawel J, Ageno W, et al. Low molecular weight heparin for the treatment of retinal vein occlusion: a systematic review and meta-analysis of randomized trials. Haematologica 2010;95:1587-93.

64. Squizzato A, Manfredi E, Bozzato S, et al. Antithrombotic and fibrinolytic drugs for retinal vein occlusion: a systematic review and a call for action. Thromb Haemost 2010;103:271-6.

65. Pielen $\mathrm{A}$, Feltgen $\mathrm{N}$, Isserstedt $\mathrm{C}$, et al. Efficacy and safety of intravitreal therapy in macular edema due to branch and central retinal vein occlusion: a systematic review. PLOS ONE 2013;8:e78538 